\title{
Multiplicity in T and OB Associations
}

\author{
Rainer Köhler \\ Astrophysikalisches Institut Potsdam, An der Sternwarte 16, D-14482 \\ Potsdam, Germany \\ Wolfgang Brandner \\ University of Hawaii, Institute for Astronomy, 2680 Woodlawn Dr., \\ Honolulu, HI 96822, USA
}

\begin{abstract}
The results of binary surveys among $\mathrm{T}$ Tauri stars in the $\mathrm{T}$ associations Taurus-Auriga and Chamaeleon, and in the OB association Scorpius-Centaurus are summarized, and implications on our understanding on the formation of binary and multiple systems are discussed.
\end{abstract}

\section{Introduction}

In 1993, two studies gave independently from each other the surprising result that $\mathrm{T}$ Tauri stars in the Taurus-Auriga star-forming region have nearly twice as many companions as their older counterparts on the main sequence (Leinert et al. 1993, Ghez et al. 1993). Extrapolated to the full range of orbital periods, this means that all or nearly all young stars are binary or multiple systems. This leads to the insight that star formation always means binary formation, which in turn is one of the reasons for this conference. While other studies also found a high multiplicity among young stars (e.g. Simon et al. 1995, Ghez et al. 1997), newer surveys indicate that things are more complicated and that it is not sufficient to use the fraction of binaries or multiples to describe the binary population in a given star-forming region.

\section{T Associations}

\subsection{Taurus-Auriga}

One explanation for the overabundance of young binary systems is that the samples studied may not be representative because they mainly contain classical T Tauri stars (CTTS). According to the ROSAT survey, however, the majority of young stars are X-ray emitting weak-line T Tauri stars (WTTS). To test this hypothesis we conducted a survey of 76 stars in Taurus-Auriga (Köhler and Leinert 1998). These stars were discovered with the help of ROSAT and identified as T Tauri stars by Wichmann et al. (1996). Combined with the list of 104 stars of Leinert et al. (1993), this gives a sample of 180 stars.

Figure 2 shows the distribution of periods of these stars compared to that of main-sequence stars (Duquennoy \& Mayor 1991). We find (54.2 \pm 8.7 ) com- 

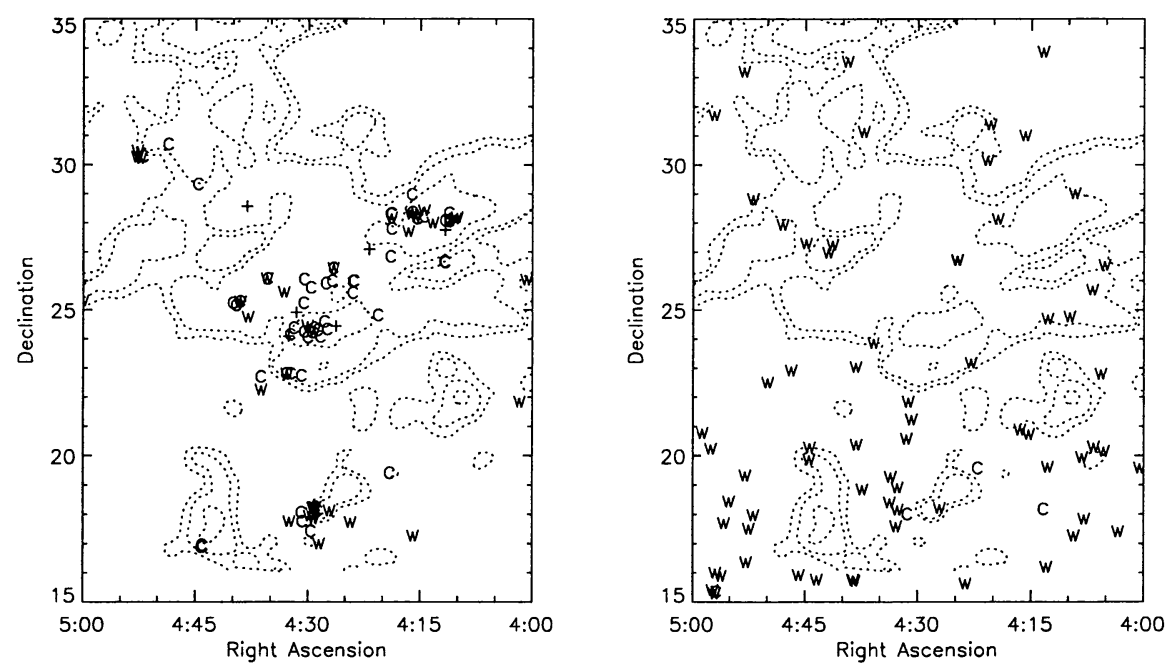

Figure 1. Spatial distribution of $\mathrm{T}$ Tauri stars known prior to ROSAT (left panel) and the new T Tauri stars discovered by Wichmann et al. 1996, right panel). Our combined sample consists of the stars in the left plus the stars in the right panel. Classical T Tauri stars are marked by "C", weak-line T Tauri stars by "W", and unclassified $\mathrm{T}$ Tauri stars by crosses. The dotted contours represent the integrated CO J=1-0 line brightness (Ungerechts \& Thadeus 1987).

panions per 100 CTTS, and (44.1 16.6$)$ companions per 100 WTTS. This means the binary frequency of WTTS is within the errors as high as that of CTTS. A $\chi^{2}$-test shows that also the period distributions are essentially identical.

Duquennoy \& Mayor (1991) found (25.3 \pm 3.9$)$ companions per 100 mainsequence stars in the range of periods sampled by our survey. This corresponds to an excess of pre-main-sequence multiples by a factor of $(1.93 \pm 0.26)$ compared to the main sequence.

Another important statistical quantity of binaries is the distribution of flux ratios, which is shown on the right hand side of figure 2 . We find that close companions have a flat brightness ratio distribution with a tendency towards equal brightnesses, while wide binaries preferentially have low brightness ratios. If we take the flux ratios in $\mathrm{K}$ as an approximation for the mass ratios, then our findings match the prediction of the model calculations of Bate and Bonnell (Bate 1997, Bate \& Bonnell 1997).

\subsection{Chamaeleon}

The Chamaeleon star-forming region is a $\mathrm{T}$ association similar to Taurus-Auriga. Some 17 stars were surveyed for binaries by Ghez et al. (1997). With the help of the ROSAT All-Sky Survey, 80 new T Tauri stars have been discovered by Alcalá et al. (1995). 

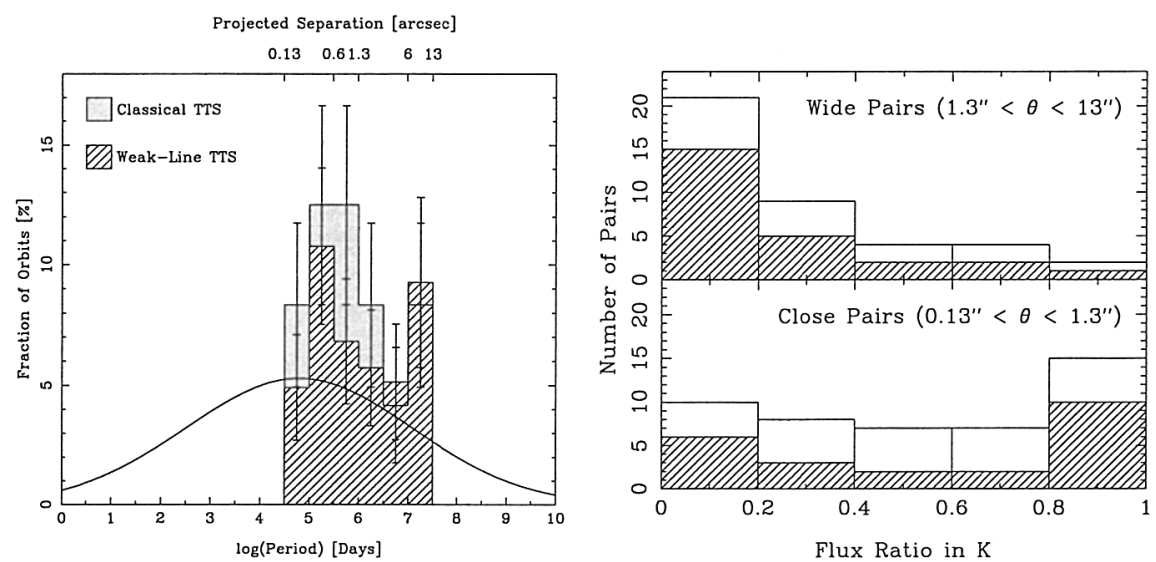

Figure 2. Left: Binary frequency in Taurus-Auriga as a function of orbital period and separation, plotted separately for classical (shaded histogram) and weak-line $\mathrm{T}$ Tauri stars (hatched histogram). By binary frequency we mean the number of companion stars with orbital period in a given interval divided by the total number of systems. The Gaussian-shaped curve is the distribution of binaries among solar-type main-sequence stars (Duquennoy \& Mayor 1991).

Right: Distribution of flux ratios for close companions (between $0.13^{\prime \prime}$ and $1.3^{\prime \prime}$ or $18 \mathrm{AU}$ and $180 \mathrm{AU}$ separated from the primary) and for distant companions (between $1.3^{\prime \prime}$ and $13^{\prime \prime}$ or $180 \mathrm{AU}$ and $1800 \mathrm{AU}$ from the primary). The hatched histogram shows the numbers of companions to WTTS, the open histogram those of companions to CTTS.

We conducted a binary survey of these stars, using speckle interferometry in the K-band (Köhler et al., in prep.). We find a significant difference between stars known before and stars discovered by ROSAT. While the binary frequency in the sample of Ghez et al. (1997) is enhanced by a factor of more than three compared to the main sequence, the number of binaries we find among X-ray selected stars is even lower than among main-sequence stars.

This would support the hypothesis that the X-ray selected stars, which are spread over a much larger region than the stars known before ROSAT, are so-called run-away $\mathrm{T}$ Tauri stars. Run-away $\mathrm{T}$ Tauri stars were ejected from systems of a few stars. Therefore, they should be single stars or close binaries. However, proper motion studies show no sign that the X-ray selected stars move away from the cloud cores (Frink et al. 1998).

\section{OB Associations}

\subsection{OB Associations: Why and Where?}

- Why study OB associations? - T assocations are not necessarily the typical birthplaces for field stars as up to $\mathbf{8 0 \%}$ of all field stars might originate 


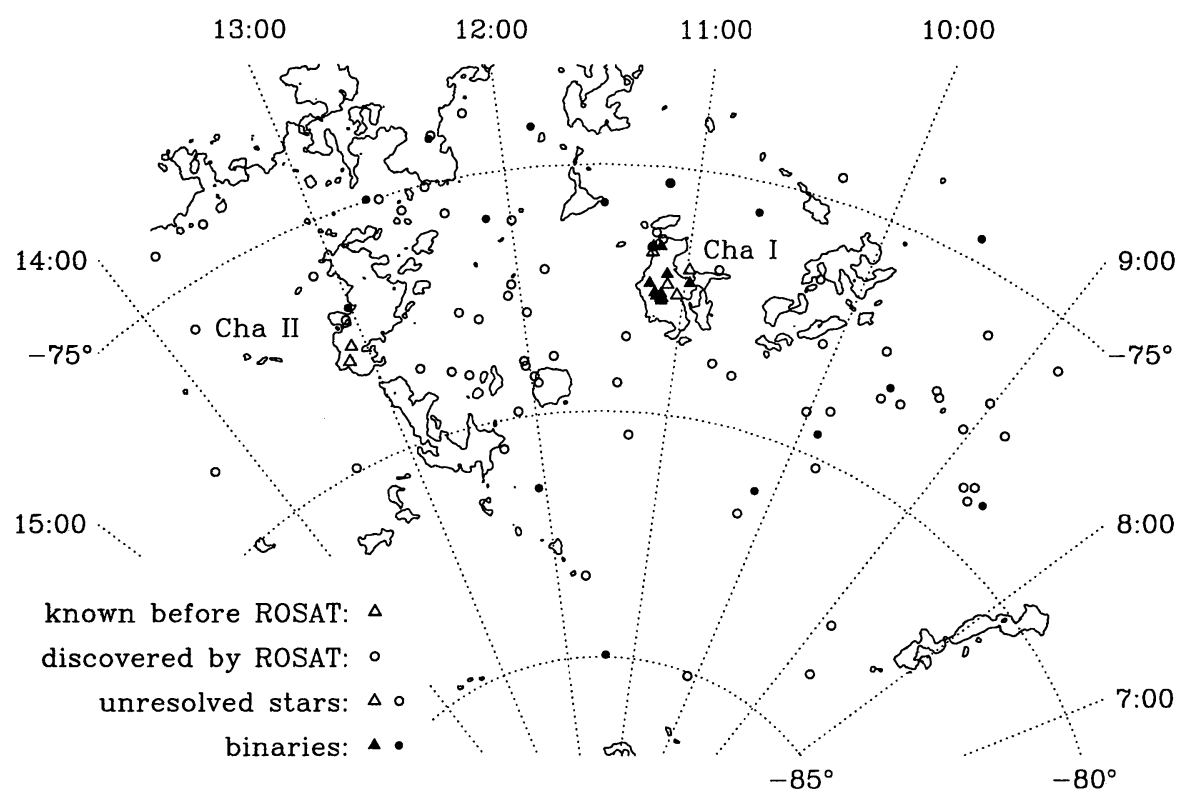

Figure 3. Spatial distribution of T Tauri stars in Chamaeleon. Stars known before ROSAT are marked by triangles, those discovered with the help of ROSAT by circles. Open symbols denote unresolved stars, filled symbols denote binaries.

in OB associations (Miller \& Scalo 1978). This naturally leads to the question whether different star forming regions have different binary fractions or not.

- Where are they located? - There are at least $15 \mathrm{OB}$ associations located within $1.5 \mathrm{kpc}$ of the Sun (Blaauw, 1991). Scorpius-Centaurus at a distance of $150 \mathrm{pc}$ is the most nearby $\mathrm{OB}$ association.

\subsection{Scorpius-Centaurus: How Do Binaries Form (and Evolve)?}

Because of its proximity, the Scorpius-Centaurus OB association and its individual subgroups can be studied in greater detail than any other OB association. Stellar population studies facilitate a reconstruction of the sequence of events (e.g., Blaauw 1991):

- more than $15 \mathrm{Myr}$ ago: existence of a Giant Molecular Cloud $\left(\mathrm{M} \geq 10^{5} \mathrm{M}_{\odot}\right)$

- 15 to 12 Myr ago: formation of stars in the Upper Centaurus-Lupus subgroup

- 12 to 10 Myr ago: formation of stars in the Lower Centaurus-Crux subgroup

- 7 to 5 Myr ago: formation of stars in the Upper Scorpius subgroup

- starting $1 \mathrm{Myr}$ ago: low-mass star formation in the Ophiuchus molecular cloud and in Lupus $\mathrm{T}$ association

The Scorpius-Centaurus OB association shows clear evidence for sequential (though not necessarily triggered) star formation. The only remnants of the once giant molecular cloud are the dark clouds in Lupus and Ophiuchus. The 


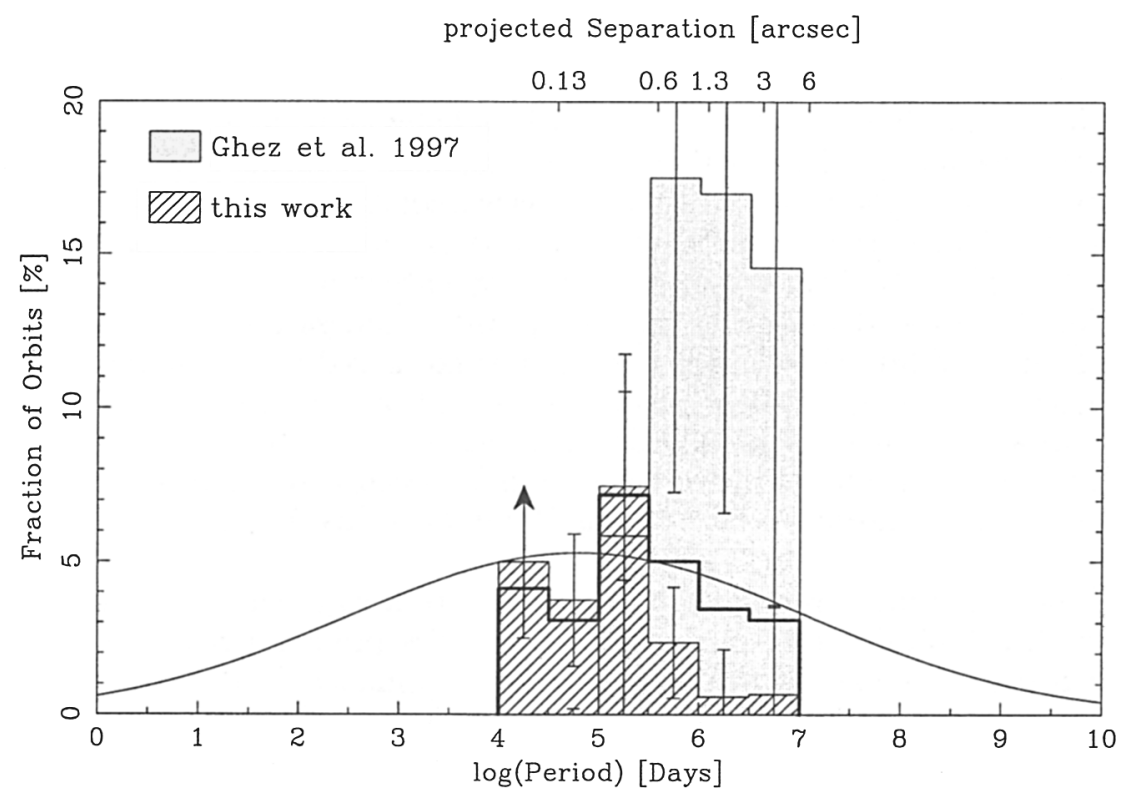

Figure 4. Binary frequency in Chamaeleon as a function of orbital period and separation, plotted separately for stars known before ROSAT (Ghez et al. 1997, shaded histogram) and stars discovered by ROSAT (hatched histogram). The thick line shows the distribution of the combination of both samples. The Gaussian-shaped curve is the distribution of binaries among solar-type main-sequence stars (Duquennoy \& Mayor 1991).

information on the initial physical conditions in the giant molecular cloud has been lost.

A number of recent studies focus on Upper Scorpius, the youngest of the major subgroups of the OB association. X-ray surveys with HEAO2 and ROSAT and ground-based follow-up studies led to the identification of $\approx 120$ low-mass pre-main sequence stars (Walter et al. 1994, Kunkel et al. 2001). At an age of 5 to $7 \mathrm{Myr}$ classical T Tauri stars are no longer present and the low-mass stellar population of Upper Scorpius no longer exhibits strong accretion signatures.

\subsection{Results of Binary Surveys}

Various methods have been applied to search for binary and multiple systems among the low-mass pre-main sequence stars in Upper Scorpius. Walter et al. (1994) found four spectroscopic binaries in a sample of 20 stars. Kunkel et al. (2001) identified eleven spectroscopic binaries in a sample of 71 stars. Speckle surveys by Ghez et al. (1993) and Köhler et al. (2000) led to the identification of 11 binaries among 24 stars, and 39 binaries among 118 stars, respectively. Using direct imaging, Brandner et al. (1996) found twelve binaries among 74 stars, and a survey for substellar companions using the Hubble Space Telescope 
and NICMOS led to the identification of five binaries in a sample of eleven stars (Brandner et al. 2000).

The surveys indicate that for separations between $0.15^{\prime \prime}$ to $6^{\prime \prime}$ (22 A.U. to 900 A.U.) the multiplicity is higher than among main-sequence stars $(\mathrm{P}=32000$ days to $10^{7}$ days) by a factor of 1.6 . The binary frequencies in the Upper Scorpius $\mathrm{OB}$ association and the Taurus $\mathrm{T}$ association thus appear to be comparable, and they are significantly higher than for main-sequence stars.

The flux ratio distribution between primary and secondary is essentially flat, which could hint that binary formation is not based on "Random pairing". No objects with extreme mass ratios were found, and in particular no young brown dwarf companions have been identified so far. Interestingly, though, the distribution of binary separations differs between two subregions of Upper Scorpius (in the following referred to as Upper Scorpius A and Upper Scorpius B):

Upper Scorpius A is centred on a region with a large number of early type stars with a typical age of $5 \mathrm{Myr}$. The low-mass pre-main sequence stars in Upper Scorpius A exhibit a higher Lithium abundance (and thus possibly a younger age) than their counterparts in Upper Scorpius B. Low-mass pre-main sequence stars in Upper Scorpius A tend to form closer visual binaries.

Upper Scorpius B houses less early type stars, and is actually located at the interface between Upper Scorpius and the older Upper Centaurus-Lupus subgroup. The age spread of the young stars in Upper Scorpius B thus very likely ranges from 5 to $15 \mathrm{Myr}$. The low-mass pre-main sequence stars exhibit a lower Lithium abundance and are thus probably older than their counterparts in Upper Scorpius A. The visual binaries identified in Upper Scorpius B are wider than in Upper Scorpius A (see Figure 5).

Thus the initial question "Do different star forming regions have different binary fractions?" transforms into "Do binary populations in different star forming regions have different period (separation) distributions?'

\subsection{Binary Properties: A Fossil Record of the Initial Physical Con- ditions in a Molecular Cloud?}

Various initial physical conditions might affect the formation of binary and multiple systems:

- The temperature of molecular clouds cores might influence binary properties (Durisen \& Sterzik 1994): "Binary Formation is more likely in low-temperature clouds"

- Internal turbulence and the angular momentum can determine binary properties (Bodenheimer et al. 2000, PPIV).

- The critical density at which the magnetic field decouples from the matter defines the binary period: $\tau_{b} \propto n_{c r}^{-2}$ (Mouschovias 1977; Boss 1997)

Unfortunately, by the time low-mass pre-main sequence stars become accessible for binary studies in the optical and near infrared, the initial giant molecular clouds are long since gone. It is thus hard (impossible?) to determine the initial conditions AFTER the stars have formed. 

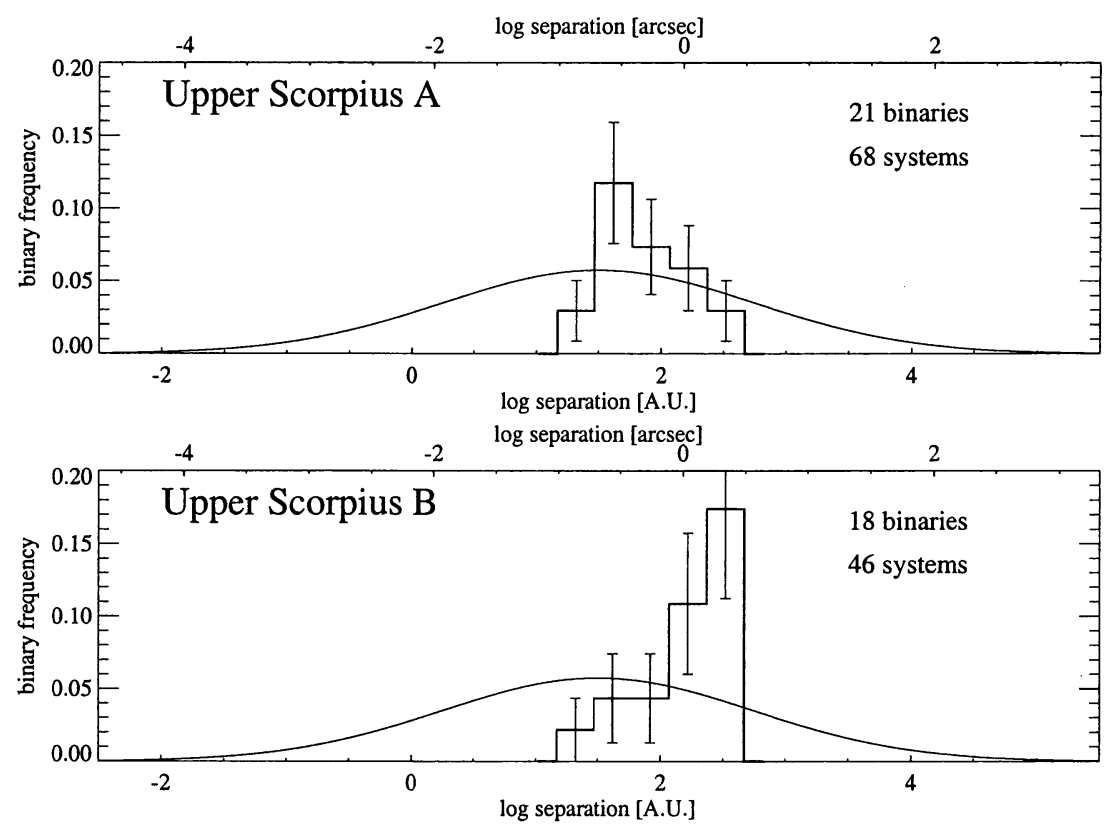

Figure 5. Histograms of the distribution of binary star separations for weak-line T Tauri stars in Upper Scorpius A and B. The lognormal type distribution found by Duquennoy \& Mayor (1991) for main-sequence stars is shown as well.

\section{Summary: What Have We Learned?}

- The Binary Frequency in $\mathrm{T}$ and $\mathrm{OB}$ associations appears to be higher than among main-sequence stars in the field. This indicates that a large fraction of the field stars might not originate in associations, but in cluster environments. - The distribution of Brightness Ratios between primary and secondary appears to be "flat". Binary stars apparently do not form by "random pairing".

- The Physical Properties of Binary Stars (and the binary frequency?) appear to reflect the initial physical conditions (density, temperature, turbulence, magnetic field?) in the parental molecular cloud

- High-spatial resolution observations of the earliest stages in the formation of binary stars are needed. In the near future, the Sub-Millimeter Array (SMA) and the Atacama Large Millimeter Array (ALMA) will enable detailed studies of the earliest stages of (binary) star formation.

\section{References}

Alcalá, J. M., Krautter, J., Covino, E., Neuhäuser, R., Schmitt, J. H. M. M., Wichmann, R. 1996, A\&A, 319, 184

Bate, M. R. 1997, MNRAS, 285, 16 
Bate, M. R., Bonnell, I. A. 1997, MNRAS, 285, 33

Blaauw, A. 1991, in The Physics of Star Formation and Early Stellar Evolution, ed. C. J. Lada \& N. D. Kylafis

Bodenheimer, P., Burkert, A., Klein, R. I., Boss, A. P. 2000, Protostars and Planets IV, ed. V. Mannings, A. P. Boss \& S. S. Russell, 675

Boss, A. P. 1997, ApJ, 483, 309

Brandner, W., Köhler, R. 1998, ApJ, 499, L79

Brandner, W., Alcalá, J. M., Kunkel, M., Moneti, A., Zinnecker, H. 1996, A\&A, 307,121

Brandner, W., Zinnecker, H., Alcalá, J. M., Allard, F., Covino, E., Frink, S., Köhler, R., Kunkel, M., Moneti, A., Schweitzer, A. 2000, AJ, in press (August issue)

Duquennoy, A., Mayor, M. 1991, A\&A, 248, 455

Durisen, R., Sterzik, M. 1994, A\&A, 286, 84

Frink, S., Röser, S., Alcalá, J. M., Covino, E., Brandner, W. 1998, A\&A, 338, 442

Ghez, A. M., Neugebauer, G., Matthews, K. 1993, AJ, 106, 2005

Ghez, A. M., McCarthy, D. W., Patience, J. L., Beck, T. L. 1997, ApJ, 481, 378

Köhler, R., Leinert, Ch. 1998, A\&A, 331, 977

Köhler, R., Kunkel, M., Leinert, Ch., Zinnecker, H. 2000, A\&A, 356, 541

Kunkel, M., Brandner, W., Yorke, H. W., Zinnecker, H. et al. 2001, in prep.

Leinert, Ch., Zinnecker, H., Weitzel, N., Christou, J., Ridgway, S. T., Jameson, R., Haas, M., Lenzen, R. 1993, A\&A, 278, 129

Miller, G. E., Scalo, J. M. 1978, PASP, 90, 506

Mouschovias, T. Ch. 1977, ApJ, 211, 147

Simon, M., Ghez, A. M., Leinert, Ch., Cassar, L., Chen, W. P., Howell, R. R., Jameson, R. F., Matthews, K., Neugebauer, G., Richichi, A. 1995, ApJ, 443,625

Ungerechts, H., Thaddeus, P. 1987, ApJS, 63, 645

Walter, F. M., Vrba, F. J., Mathieu, R. D., Brown, A., Myers, P. C. 1994, AJ, 107,692

Wichmann, R., Krautter, J., Schmitt, J. H. M. M., Neuhäuser, R., Alcalá, J. M., Zinnecker, H., Wagner, R. M., Mundt, R., Sterzik, M. F. 1996, A\&A, 312,439 